\title{
Increased expression of cyclooxygenase-2 in synovium tissues and synovial fluid from patients with knee osteoarthritis is associated with downregulated microRNA-758-3p expression
}

\author{
ZHEN LIU $^{1}$, JING SUN ${ }^{2}$, TINGTING LIANG ${ }^{3}$ and XIAONAN HUANG ${ }^{4}$ \\ Departments of ${ }^{1}$ Joint Surgery and ${ }^{2}$ Surgical Nursing, Heze Municipal Hospital, Heze, \\ Shandong 274000; ${ }^{3}$ Department of Surgical Nursing, Dongda Hospital of Shanxian County, Heze, \\ Shandong 274300; ${ }^{4}$ Department of Hand and Foot Microsurgery, \\ Heze Municipal Hospital, Heze, Shandong 274000, P.R. China
}

Received July 14, 2020; Accepted February 26, 2021

DOI: $10.3892 /$ etm.2021.10433

\begin{abstract}
Cyclooxygenase-2 (COX-2) is a common factor in inflammation, and its specific regulatory mechanism has not been fully elucidated. The present study aimed to investigate COX-2 mRNA and protein expression levels in synovium tissues and synovial fluid from patients with knee osteoarthritis (KOA), and determine the molecular mechanism by which microRNA (miRNA/miR)-758 regulates KOA via COX-2. A total of 37 patients with KOA and 29 patients with acute knee trauma (control group) were enrolled in the present study. Reverse transcription-quantitative PCR analysis was performed to detect miR-758-3p and COX-2 mRNA expression, while western blotting and ELISA were performed to detect COX-2 protein expression in synovium and synovial fluid, respectively. The dual-luciferase reporter assay was performed to verify the interaction between miR-758-3p and the 3'-untraslated region (UTR) of COX-2 mRNA. Synovial cells were transfected with agomiR-758-3p, and the MTT assay was performed to assess cell proliferation. The results demonstrated that COX-2 expression was higher in patients with KOA than those with acute knee trauma. Conversely, miR-758-3p expression was lower in patients with KOA than those with acute knee trauma. Notably, miR-758-3p interacted with the 3'-UTR of COX-2 mRNA to regulate its expression. Overexpression of miR-758-3p inhibited the expression and release of COX-2, as well as the proliferation of human KOA synovial cells. Taken together, these results suggest that COX-2 expression is upregulated in synovium tissues and synovial fluid from patients with KOA, which is associated
\end{abstract}

Correspondence to: Dr Xiaonan Huang, Department of Hand and Foot Microsurgery, Heze Municipal Hospital, 2888 Caozhouxi Road, Heze, Shandong 274000, P.R. China E-mail:hfiauo@163.com

Key words: knee osteoarthritis, microRNA-758-3p, cyclooxygenase-2, synovium, synovial fluid with downregulated miR-758-3p expression. In addition, miR-758-3p affects the proliferation of synovial cells and the expression of relevant proteins in these cells, thus promoting the occurrence and development of KOA.

\section{Introduction}

Knee osteoarthritis (KOA) is a disease characterized by severe wear of articular cartilage and regeneration of articular margin and subchondral bone (1). Similar to hip OA, KOA can cause weight-bearing difficulty in the lower limbs, eventually resulting in limb disability (2). Inflammatory response induced by synovium tissue injury is an common cause of OA $(3,4)$. The main symptoms of KOA include joint pain and stiffness (5). Joint pain initially appears in joint movement and gradually develops into persistent pain (5). The incidence and development of KOA are associated with age, and the incidence rate is significantly higher in women compared with men (6). According to the etiology and pathogenesis of KOA, it is divided into two subtypes, primary and secondary KOA (7). The pathological process of primary KOA is gradual, and its pathogenesis remains unclear. Secondary KOA often occurs in young adults, and common causes include accidental trauma, deformity, insufficiency of blood supply and joint inflammation (7). Notably, inflammatory mediators, proteoglycans and matrix metalloproteinases (MMPs) are all involved in the pathogenesis of KOA (8-10).

Cyclooxygenase-2 (COX-2) is a member of the cyclooxygenase isoenzyme family, which catalyzes the metabolism of arachidonic acid to form prostaglandins (PGEs). PGE2, an inflammatory mediator, can activate inositol triphosphate through its receptors (EP1R, EP2R, EP3R or EP4R) to promote the release of $\mathrm{Ca}^{2+}$ and inhibit $\mathrm{Na}^{+} / \mathrm{Ca}^{2+}$ exchange channel to decrease $\mathrm{Ca}^{2+}$ outflow (11). Simultaneously, intracellular $\mathrm{Ca}^{2+}$ release elevates nitrogen oxides expression, which induces the production of reactive oxygen species (ROS) and oxidative stress $(12,13)$. COX-2 expression increases with the occurrence of inflammatory reaction and oxidative stress, which in turn increases the expression levels of the inflammatory mediators, PGE2 
and PGH2, as well as vascular endothelial growth factor, which may be induced by PGE2 (14). Thus, the production of oxidative stress-related substances, such as oxygen free radicals and ROS is indirectly induced, further promoting cell damages $(14,15)$. In addition, COX-2 is closely associated with inflammation-related diseases and tumors, and it is speculated that COX-2 can promote tumor angiogenesis, tissue invasion and enhance the apoptosis resistance of tumor cells (16-18). However, the regulation of COX-2 remains unclear.

MicroRNA (miRNA/miR)-758 has been demonstrated to play important regulatory roles in cell metabolism, cholesterol outflow, atheromatous plaque formation and hepatitis C virus (HCV) infection (19). For example, miR-758-3p can regulate cholesterol efflux by inhibiting ABCA1 protein expression (20). Furthermore, Mandolini et al (21) reported that miR-758-3p regulates ABCA1 expression in human atherosclerotic plaques. Yang et al (19) demonstrated that miR-758-3p expression is significantly upregulated in HCV infection. In addition, abnormal miR-758-3p expression is associated with tumor progression (22). During chemotherapy for esophageal cancer, chemotherapeutic drugs can inhibit miR-758-3p expression, and the loss of control of miR-758-3p to its downstream target genes may be the reason for drug resistance of tumors (23). The bioinformatics of the present study predicted that miR-758-3p has the potential to regulate COX-2 expression. However, the underlying molecular mechanism by which miR-758-3p regulates COX-2 has not yet been investigated.

The present study aimed to investigate COX-2 mRNA and protein expression levels in synovium tissues and synovial fluid from patients with KOA, and determine the underlying molecular mechanism by which miR-758-3p regulates KOA via COX-2.

\section{Materials and methods}

Patients. A total of 37 patients with KOA who received treatment at Heze Municipal Hospital between December 2017 and July 2019 were included in the experimental group (20 men and 17 women; age range, $30-60$ years; median age, 47.6 years). A total of 29 patients with acute knee trauma who received treatment at Heze Municipal Hospital during the same period were included in the control group (17 men and 12 women; age range, 28-58 years; median age, 46.9 years). All patients with KOA met the 2010 EULAR recommendations (24) for diagnosis. Patients with cancer, rheumatoid arthritis and other immune diseases were excluded from the present study. Synovium tissue samples were collected from patients with KOA during knee replacement or knee arthroscopy, while synovium tissues were collected from patients with knee trauma by resection during surgery. Part of the synovium was used to culture primary cells, while part was used for detection. Synovial fluid was respectively collected from patients with KOA and knee trauma during surgery, and blood cells were removed via centrifugation at $1,000 \mathrm{xg}$ at $4^{\circ} \mathrm{C}$ for $10 \mathrm{~min}$. All samples were stored at $-80^{\circ} \mathrm{C}$ until subsequent experimentation. The present study was approved by the Ethics Committee of Heze Municipal Hospital (Heze, China; approval no. 20161226-85) and written informed consent was provided by all patients or their families prior to the study start.
Reverse transcription-quantitative (RT-q)PCR. Total RNA was extracted from fluid and tissue samples using TRIzol ${ }^{\circledR}$ reagent (cat. no. R0016; Beyotime Institute of Biotechnology), according to the manufacturer's instructions. The purity of RNA was assessed by measuring A260/A280 on a reader. According to the manufacturer's protocol, mRNA or miRNA $(2 \mu \mathrm{g})$ was reverse-transcribed into cDNA at $42^{\circ} \mathrm{C}$ using the TIAN Script II cDNA First Strand Synthetic kit and miRcute miRNA cDNA First Strand Synthetic kit, respectively (both purchased from Tiangen Biotech Co., Ltd.). The following primer sequences were used for qPCR using SYBR-Green (cat. no. Q121-02; Vazyme Biotech Co., Ltd.): COX-2 forward, 5'- CAGCCATACAGCAAATCCTTG-3' and reverse, 5'- CAA ATGTGATCTGGATGTCAAC-3'; GAPDH forward, 5'-TGA CCTTGCCCACAGCCTTG-3' and reverse, 5'-CATCAC CATCTTCCAGGAGCG-3'; miR-758-3p forward, 5'-ACA CTCCAGCTGGGTTTGTGACCTGGTCCA-3' and reverse, 5'-TGGTGTCGTGGAGTCG-3'; and U6 forward, 5'-CTCGCT TCGGCAGCACA-3' and reverse, 5'-AACGCTTCACGAATT TGCGT-3'. The following thermocycling conditions were used for qPCR: Initial denaturation at $95^{\circ} \mathrm{C}$ for $5 \mathrm{~min}$; denaturation at $95^{\circ} \mathrm{C}$ for $30 \mathrm{sec}$, annealing at $55^{\circ} \mathrm{C}$ for $30 \mathrm{sec}$ and elongation at $72^{\circ} \mathrm{C}$ for $60 \mathrm{sec}(35 \mathrm{cycles})$; final extension at $72^{\circ} \mathrm{C}$ for $2 \mathrm{~min}$. Relative expression levels were calculated using the $2^{-\Delta \Delta \mathrm{Cq}}$ method (25). If the Cq value was too high ( $>35)$, the dose was adjusted, and the experiment was repeated to increase reliable. All experiments were performed in triplicate.

Cell culture. 293T cells were purchased from The Cell Bank of Type Culture Collection of the Chinese Academy of Sciences. Cells were maintained in DMEM/F-12 medium (Thermo Fisher Scientific, Inc.) supplemented with $10 \%$ fetal bovine serum (FBS; Thermo Fisher Scientific, Inc.), at $37^{\circ} \mathrm{C}$ with $5 \%$ $\mathrm{CO}_{2}$.

Prior to isolating KOA synovial cells, synovium tissues were washed with PBS containing $2 \%$ penicillin-streptomycin (Thermo Fisher Scientific, Inc.). Tissue samples were cut into $1-\mathrm{mm}^{3}$-thick sections and digested using $4 \mathrm{mg} / \mathrm{ml}$ type II collagenase (Thermo Fisher Scientific, Inc.) at $37^{\circ} \mathrm{C}$ for $4 \mathrm{~h}$. The solution was subsequently diluted with PBS, filtered through 200 -mesh sterile sieves and centrifuged at $37^{\circ} \mathrm{C}$ and $800 \mathrm{x} \mathrm{g}$ for $5 \mathrm{~min}$. Cells were resuspended in DMEM supplemented with $20 \%$ FBS and $1 \%$ penicillin-streptomycin at $37^{\circ} \mathrm{C}$ with $5 \% \mathrm{CO}_{2}$. Synovial cells are generally stable within 5 passages (26), thus cells at passages 3-7 were used for subsequent experimentation.

Cells at the logarithmic phase $\left(3 \times 10^{5}\right)$ were seeded into 24-well plates and maintained in antibiotic-free DMEM/F-12 medium supplemented with $10 \%$ FBS $24 \mathrm{~h}$ prior to transfection, until they reached $70 \%$ confluence. In the first vial, $1 \mu 1$ miR-NC (20 pmol $/ \mu 1$; forward, 5'-UUCUCCGAACGUGUC ACGUTT-3' and reverse, 5'-ACGUGACACGUUCGGAGA ATT-3') or miR-758-3p mimic (20 pmol $/ \mu 1$; forward, 5'-UUU GUGACCUGGUCCACUAACC-3' and reverse, 5'-UUA GUGGACCAGGUCACAAAUU-3') was added to $50 \mu 1$ Opti-MEM ${ }^{\circledR}$ medium (Thermo Fisher Scientific, Inc.). In the second vial, $1 \mu 1$ Lipofectamine ${ }^{\circledR} 2,000$ reagent (Thermo Fisher Scientific, Inc.) was added to $50 \mu 1$ Opti-MEM ${ }^{\circledR}$ medium. Following incubation at $37^{\circ} \mathrm{C}$ for $5 \mathrm{~min}$, the vials were combined and incubated for a further $20 \mathrm{~min}$ at 
room temperature. The mixture was subsequently added to each well and incubated at $37^{\circ} \mathrm{C}$ for $6 \mathrm{~h}$. The medium was replaced with DMEM/F-12 medium supplemented with $10 \%$ FBS. Cells were collected for subsequent experimentation after $48 \mathrm{~h}$.

Western blotting. Total protein was extracted from tissues or cells using E.Z.N.A total DNA/RNA/Protein kit (Omega Bio-Tek, Inc.). Total protein was quantified using the BCA Protein Assay kit (cat. no. ab102536; Abcam) and $50 \mu \mathrm{g}$ protein/lane was separated by SDS-PAGE on a $10 \%$ gel electrophoresis at $100 \mathrm{~V}$. The separated proteins were subsequently transferred onto polyvinylidene difluoride membranes on ice (100 V for $2 \mathrm{~h}$ ), and blocked with $5 \%$ skimmed milk at room temperature for $1 \mathrm{~h}$. The membranes were incubated with rabbit anti-human COX-2 (1:1,000; cat. no. ab15191; Abcam) and $\beta$-actin (1:5,000; cat. no. ab129348; Abcam) polyclonal primary antibodies, overnight at $4^{\circ} \mathrm{C}$. Membranes were washed three times with PBS containing Tween-20 (0.1\%) for $15 \mathrm{~min}$, and subsequently incubated with goat anti-rabbit horseradish peroxidase-conjugated secondary antibody (1:3,000; cat. no. ab6721; Abcam) for $1 \mathrm{~h}$ at room temperature. Membranes were re-washed three times with PBS containing Tween-20 (0.1\%) for $15 \mathrm{~min}$. Protein bands were visualized using the enhanced chemiluminescence detection kit (cat. no. ab65623; Abcam) and analyzed using Image lab v3.0 software (Bio-Rad Laboratories, Inc.).

ELISA. Synovial fluid was centrifuged at $1,000 \mathrm{x}$ at $4^{\circ} \mathrm{C}$ for $10 \mathrm{~min}$ and the supernatant was collected, which was used to detect protein contents according to the manufacturer's protocols (COX-2 ELISA kit; cat. no. ab267646; Abcam and Ca PGE2 ELISA kit; cat. no. CSB-E07965h; Cusabio Technology LLC).

Bioinformatics analysis. The miRanda database (http://www. microrna.org/microrna/home.do) was used to predict the genes that regulate COX-2 expression.

Dual-luciferase reporter assay. Based on bioinformatics analysis, wild-type (WT) and mutant regions of miR-758-3p in the 3'-UTR of COX-2 mRNA were chemically synthesized in vitro. The ends were joined with Spe-1 and HindIII restriction sites, and subsequently cloned into pMIR-REPORT luciferase reporter plasmids (Thermo Fisher Scientific, Inc.). Plasmids $(1 \mu \mathrm{g})$ with WT or mutant 3'-UTR sequences were co-transfected into $293 \mathrm{~T}$ cells with agomiR-758-3p (100 nM; forward, 5'-UUUGUGACCUGGUCCACU AACC-3' and reverse, 5'-UUAGUGGACCAGGUCACAAAU U-3') or agomiR-negative control (agomiR-NC; $100 \mathrm{nM}$; forward, 5'-UUCUCCGAACGUGUCACGUTT-3' and reverse, 5'-ACGUGACACGUUCGGAGAATT-3'; both purchased from Sangon Biotech Co., Ltd.) using Exfect Transfection Reagent (Vazyme Biotech Co., Ltd.). Following incubation at $37^{\circ} \mathrm{C}$ for $24 \mathrm{~h}$, cells were lysed using the dual-luciferase reporter assay kit (cat. no. E1980; Promega Corporation), according to the manufacturer's instructions, and luciferase activities were detected using the GloMax 20/20 luminometer (Promega Corporation). Firefly luciferase activity was normalized to Renilla luciferase activity.
MTT assay. Cell proliferation was assessed via the MTT assay. Following transfection, cells were seeded into 96-well plates at a density of $2 \times 10^{3}$ cells/well. Each condition was assessed in triplicate wells. At 24, 48 and $72 \mathrm{~h}$ post-transfection, $20 \mu \mathrm{l}$ MTT reagent (5 g/l; cat. no. C0009; Beyotime Institute of Biotechnology) was added to each well and incubated for $4 \mathrm{~h}$ at $37^{\circ} \mathrm{C}$. Following the MTT incubation, the purple formazan crystals were dissolved using DMSO (150 $\mu \mathrm{l} /$ well) and proliferation was subsequently analyzed at a wavelength of $490 \mathrm{~nm}$, using a microplate reader (Bio-Rad Laboratories, Inc.).

Statistical analysis. Statistical analysis was performed using SPSS 18.0 software (SPSS, Inc.). Each experiment was repeated three times. Data are presented as the mean \pm standard deviation. Unpaired Student's t-test was used to compare differences between two groups, while one-way ANOVA followed by Bonferroni post hoc test were used to compare differences between multiple groups. $\mathrm{P}<0.05$ was considered to indicate a statistically significant difference.

\section{Results}

COX-2 expression is higher in patients with KOA than those with acute knee trauma. RT-qPCR analysis was performed to detect COX-2 mRNA expression in synovium tissues and synovial fluid. In addition, western blotting and ELISA were performed to detect COX-2 protein expression in synovium tissues and synovial fluid, respectively. The results demonstrated that COX-2 mRNA and protein expression levels were significantly higher in synovium tissues from patients with KOA compared with the control group ( $\mathrm{P}<0.05$; Fig. 1A and $\mathrm{B})$. Similarly, COX-2 mRNA and protein expression levels were significantly higher in synovial fluid from patients with KOA compared with the control group $(\mathrm{P}<0.01$; Fig. $1 \mathrm{C}$ and $\mathrm{D})$. Taken together, these results suggest that COX-2 may play a regulatory role in the pathological process of KOA.

miR-758-3p expression is lower in patients with KOA than those with acute knee trauma. Bioinformatics analysis was performed to predict the genes that regulate $\mathrm{COX}-2$ expression. The results demonstrated that miR-758-3p can potentially interact with COX-2 (Fig. 2A). RT-qPCR analysis was performed to detect miR-758-3p expression in synovium tissues and synovial fluid. The results demonstrated that miR-758-3p expression was significantly lower in synovium tissues and synovial fluid from patients with KOA compared with the control group $(\mathrm{P}<0.05$ and $\mathrm{P}<0.01$; Fig. $2 \mathrm{~B}$ and $\mathrm{C}$, respectively). Collectively, these results suggest that miR-758-3p expression is downregulated in patients with KOA.

miR-758-3p interacts with the 3'-UTR of COX-2 to regulate its expression. The dual-luciferase reporter assay was performed to confirm the interaction between COX-2 mRNA and miR-758-3p. As presented in Fig. 3, the luciferase activity of 293T cells co-transfected with agomiR-758-3p and pMIR-REPORT was significantly lower in the wild-type group compared with the NC group $(\mathrm{P}<0.05)$. Conversely, no significant difference was observed in the luciferase activity of cells co-transfected with agomiR-758-3p and pMIR-REPORT between the mutant and NC groups ( $P>0.05$; Fig. 3$)$. Taken 

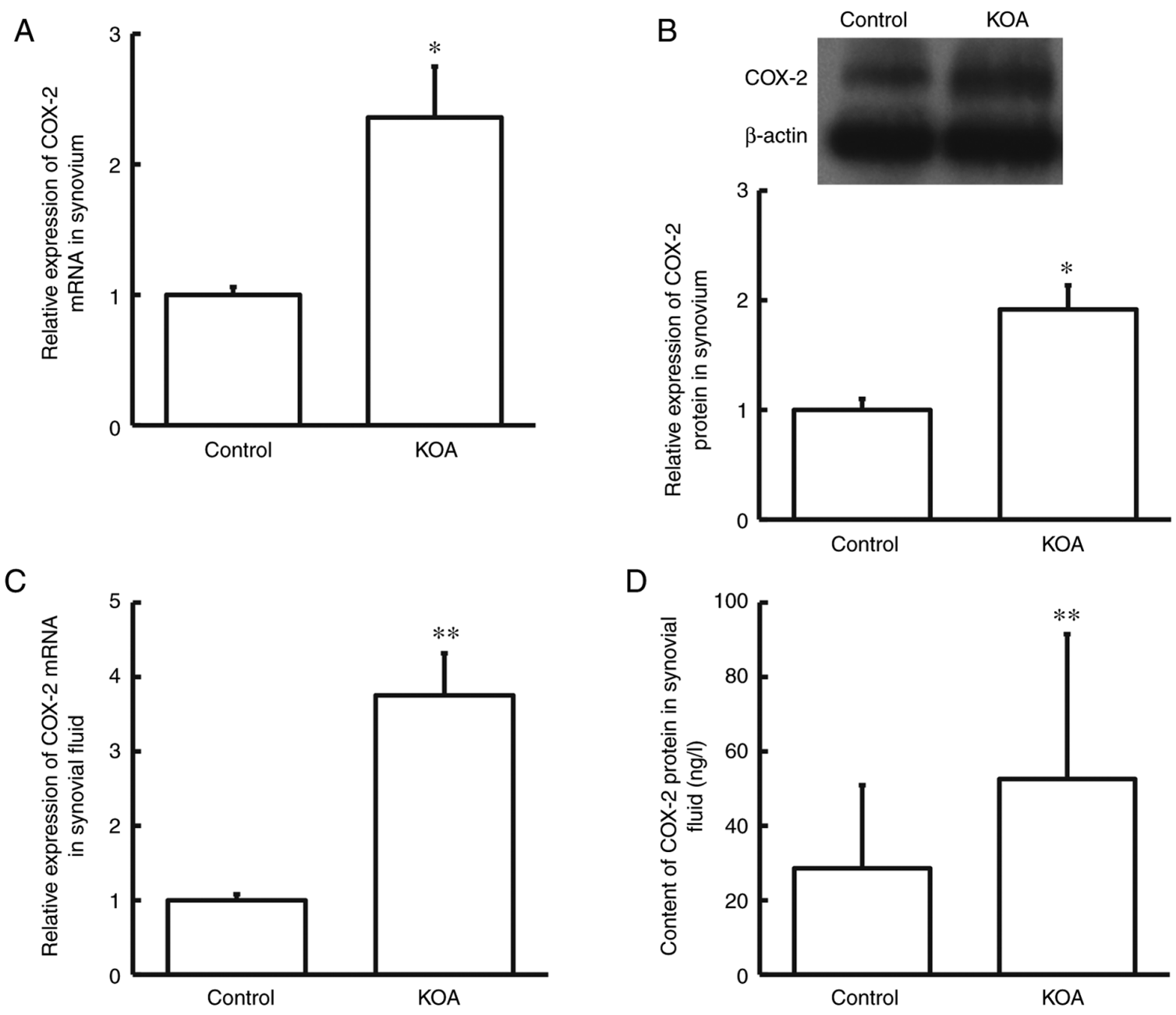

Figure 1. COX-2 expression in synovium tissues and synovial fluid. RT-qPCR and western blot analyses were performed to detect COX-2 (A) mRNA and (B) protein expression levels in synovium tissues from patients with acute knee trauma and KOA, respectively. RT-qPCR analysis and ELISA were performed to detect COX-2 (C) mRNA and (D) protein expression levels in synovial fluid from patients with acute knee trauma and KOA, respectively. ${ }^{*} \mathrm{P}<0.05$, ${ }^{* *} \mathrm{P}<0.01$ vs. control group. COX-2, cyclooxygenase-2; RT-qPCR, reverse transcription-quantitative PCR; KOA, knee osteoarthritis.

together, these results suggest that miR-758-3p interacts with the 3'-UTR of COX-2 to regulate its expression.

Overexpression of $m i R-758-3 p$ inhibits the expression and release of $C O X-2$, as well as the proliferation of human $\mathrm{KOA}$ synovial cells. To investigate how miR-758-3p regulates COX-2 expression, KOA synovial cells were transfected with agomiR-758-3p. The results demonstrated that miR-758-3p expression was significantly higher in cells transfected with agomiR-758-3p compared with the agomiR-NC group ( $\mathrm{P}<0.01$; Fig. 4A). Conversely, COX-2 mRNA and protein expression levels were significantly lower in the agomiR-758-3p group compared with the agomiR-NC group $(\mathrm{P}<0.01$ and $\mathrm{P}<0.05$; Fig. $4 \mathrm{~B}$ and $\mathrm{C}$, respectively). ELISA indicated that $\mathrm{COX}-2$ protein expression was significantly lower in the supernatant of cells transfected with agomiR-758-3p compared with the agomiR-NC group ( $\mathrm{P}<0.01$; Fig. 4D). COX-2 is also known as prostaglandin-endoperoxide synthase-2 (PTGS-2). PGE2, which is biosynthesized from arachidonic acid by COXs (PTGSs), is a physiologically active lipid (27). Notably, PGE2 protein expression was significantly lower in the supernatant of cells transfected with agomiR-758-3p compared with the agomiR-NC group
$(\mathrm{P}<0.01$; Fig. 4E). The results of the MTT assay demonstrated that cells transfected with agomiR-758-3p proliferated at a slower rate at 48 and $72 \mathrm{~h}$ compared with the agomiR-NC group $(\mathrm{P}<0.05$; Fig. 4F). Collectively, these results suggest that overexpression of miR-758-3p inhibits the expression and release of COX-2, as well as the proliferation of human KOA synovial cells.

\section{Discussion}

KOA is a chronic degenerative disease of the bone and joints. The main pathological changes of KOA are articular cartilage degeneration and hyperosteogeny (28). The main clinical symptoms of KOA include pain, swelling, limited movement, friction sound and deformity of knee joints (5). The incidence of KOA continues to increase with age and obesity, and thus has attracted great interest in clinical practice (29).

Currently, the treatment strategies for KOA lack efficiency. Western medicine is classified into drug treatment and surgical treatment (30). COX-2 is a membrane binding protein, and its expression in cartilages is elevated following impacts by physical and chemical factors, such as trauma, inflammatory 
A

5' gaaUACUUAAACACUCAGUGUUg $3^{\prime}$ mutant COX-2

I| | | || |||||||

3' ccaAUCACCUGGU-CCAGUGUUu $5^{\prime}$ 'hsa-miR-758-3p

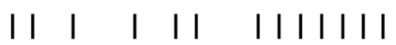

$5^{\prime}$ gaaUACUUAAACACUGUCACAAg $3^{\prime}$ wild-type COX-2
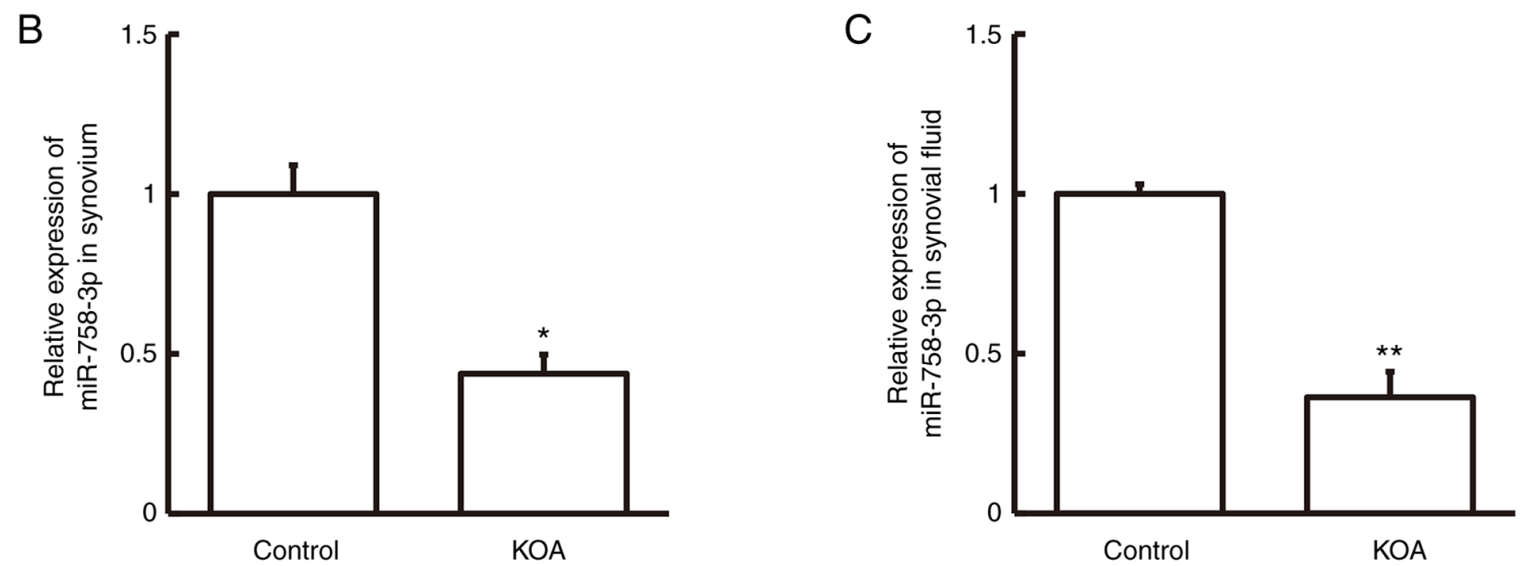

Figure 2. Bioinformatics prediction of upstream miRNA of COX-2, and miR-758 expression in synovium tissues and synovial fluid. (A) miR-758-3p can potentially interact with COX-2. Reverse transcription-quantitative PCR analysis was performed to detect miR-758-3p expression in (B) synovium tissues and $(\mathrm{C})$ synovial fluid from patients with acute knee trauma and KOA, respectively. " $\mathrm{P}<0.05$, ${ }^{* *} \mathrm{P}<0.01$ vs. control group. miRNA/miR, microRNA; COX-2, cyclooxygenase-2; KOA, knee osteoarthritis.

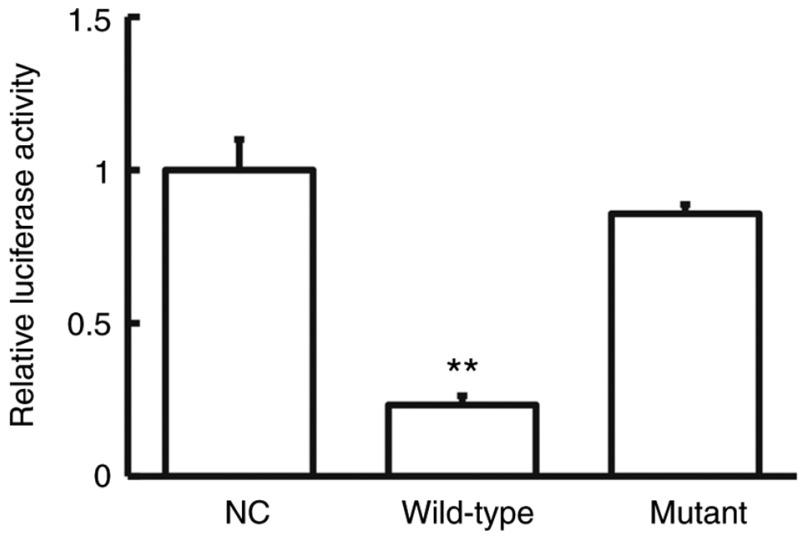

Figure 3. Relative luciferase activity of $293 \mathrm{~T}$ cells co-transfected with agomiR-758-3p and wild-type or mutant 3'-untranslated region of the COX-2 gene. The dual-luciferase reporter assay was performed to confirm the interaction between miR-758-3p and COX-2 mRNA. ${ }^{* *} \mathrm{P}<0.01$ vs. NC group. miR, microRNA; COX-2, cyclooxygenase-2; NC, negative control.

mediators and cytokines (31). COX-2 can promote the synthesis of PGE2, which induces the production of nitric oxide (32). PGE2 is a multifunctional inflammatory mediator that increases the production of MMPs and catabolic substances, affects the structure and function of the joint, and accelerates the destruction of joint structure (33). Thus, it can be used as a target for KOA therapy.

The results of the present study demonstrated that COX-2 expression was abnormally high in synovium tissues and synovial fluid from patients with KOA, suggesting that COX-2 plays an important biological role in KOA. However, the regulation of COX-2 in KOA remains unclear. Bioinformatics analysis revealed that miR-758-3p regulates COX-2 expression. miRNAs regulate mRNAs by inhibiting translation (34). It has been reported that miR-758-3p can target HMGB3 and inhibit the proliferation and metastasis of cervical cancer cells via the Wnt/ $\beta$-catenin signaling pathway (35). Similarly, miR-758-3p targets HMGA1 to inhibit the malignant phenotype of osteosarcoma via the $\mathrm{Wnt} / \beta$-catenin signaling pathway (36). miR-758-3p also inhibits the malignant progression of retinoblastoma by directly targeting PAX6 (37). In addition, miR-758-3p also inhibits lung cancer cells $(38,39)$. Based on these findings, it was speculated that miR-758-3p is closely associated with inflammation.

The results of the present study demonstrated that miR-758-3p expression was significantly lower in synovium tissues and synovial fluid from patients with KOA compared with the control group. Considering the abnormally high COX-2 expression, it was speculated that downregulated miR-758-3p expression induces upregulated COX-2 expression, which was confirmed via the dual-luciferase reporter assay. Synovial cells were subsequently transfected with agomiR-758-3p and the MTT assay was performed to assess cell proliferation. The results demonstrated that overexpression of miR-758-3p decreased COX-2 mRNA and protein expression levels, as well as the secretion of COX-2. In addition, overexpression of $\mathrm{miR}-758-3 \mathrm{p}$ decreased the proliferative ability of the cells.

The present study has a limitation in the small number of patients, which will be overcome in the future by performing studies with a larger number of patients. COX-2 is an inducible enzyme of inflammatory cytokines, including interleukin (IL)-1 and tumor necrosis factor- $\alpha$ (TNF- $\alpha$ ). Notably, IL-1 and TNF- $\alpha$ have been detected in synovial fluid from patients with KOA (40). However, the present study failed to investigate whether COX-2 expression was affected by IL-1 or TNF- $\alpha$, in addition to miR-758-3p. 
A

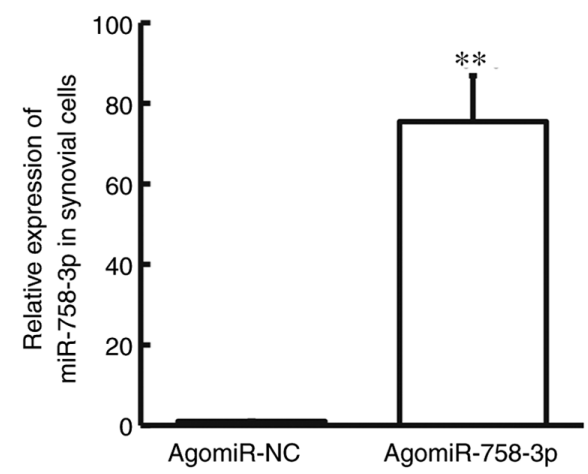

C

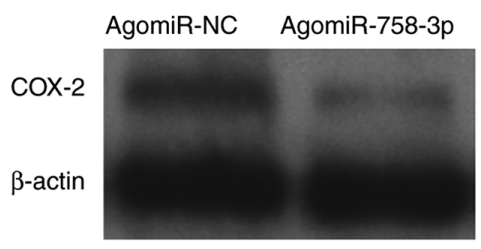

D

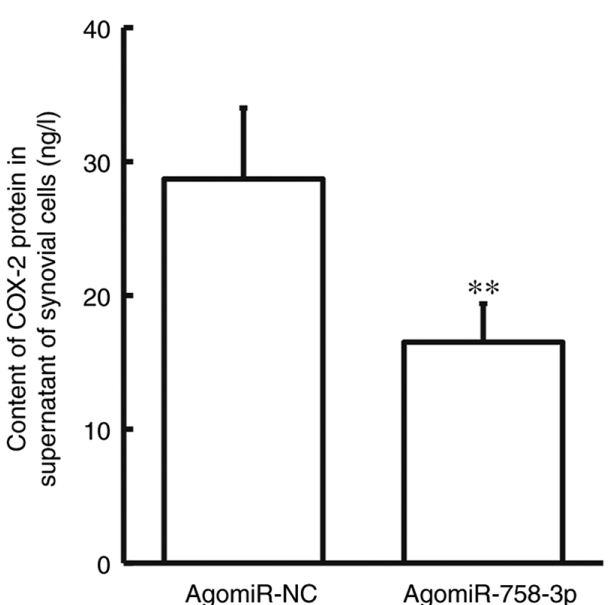

B
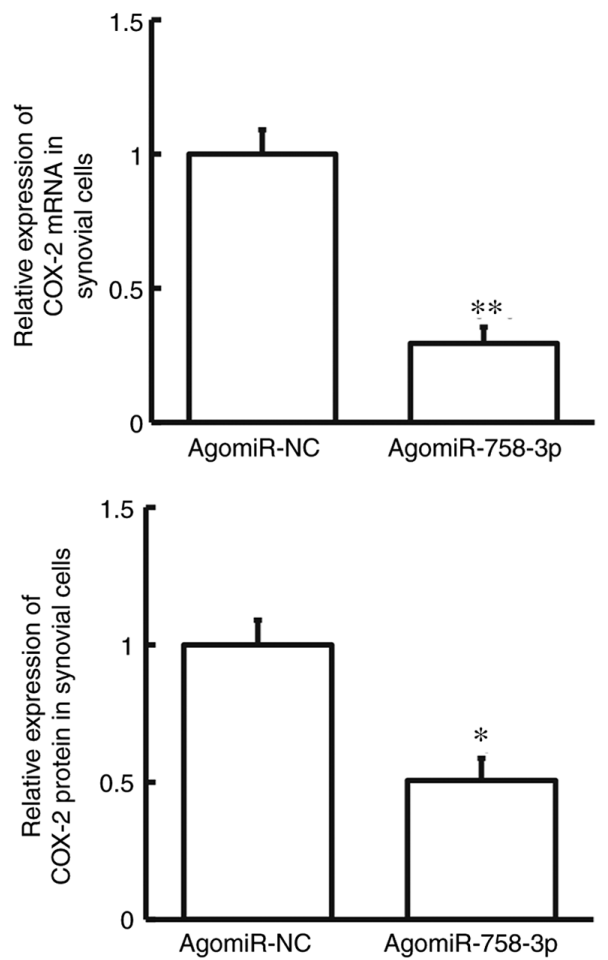

E

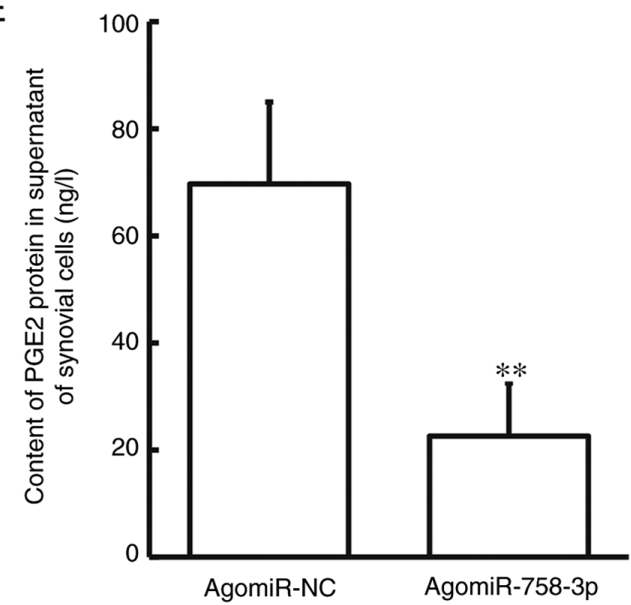

F

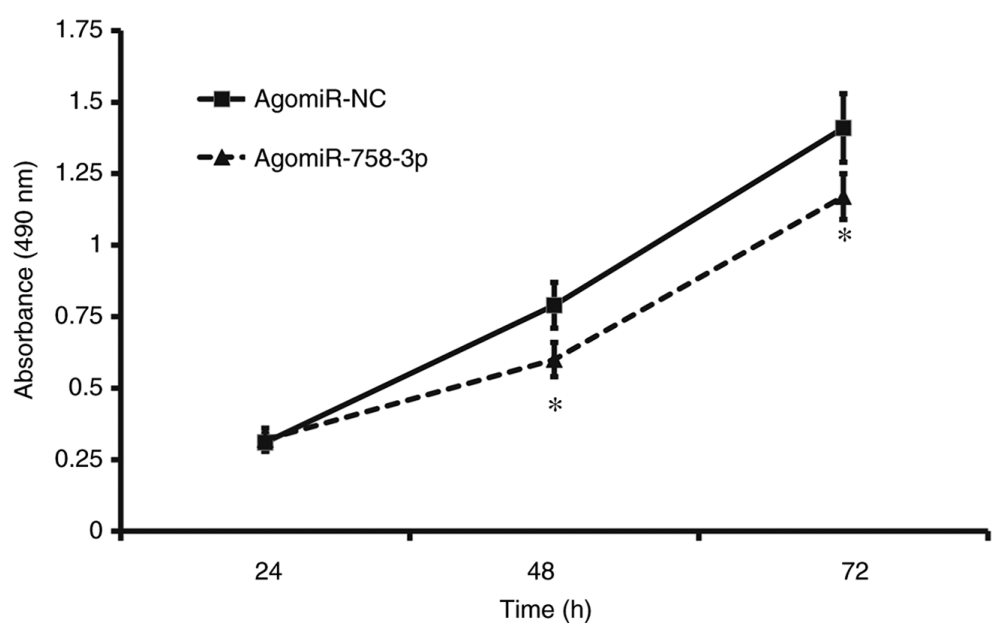

Figure 4. Effect of miR-758-3p on COX-2 expression and the proliferation of synovial cells. (A) miR-758-3p expression in synovial cells transfected with agomiR-NC or agomiR-758-3p. Reverse transcription-quantitative PCR and western blot analyses were performed to detect COX-2 (B) mRNA and (C) protein expression levels in synovial cells transfected with agomiR-NC or agomiR-758-3p, respectively. ELISA was performed to detect (D) COX-2 and (E) PGE2 protein expression levels in the supernatant of synovial cells transfected with agomiR-NC or agomiR-758-3p, respectively. (F) The MTT assay was performed to assess the proliferation of synovial cells transfected with agomiR-NC or agomiR-758-3p. $\mathrm{P}<0.05,{ }^{\text {** }} \mathrm{P}<0.01$ vs. agomiR-NC group. miR, microRNA; COX-2, cyclooxygenase-2; NC, negative control; PGE2, prostaglandin E2. 
In conclusion, the results of the present study demonstrated that miR-758-3p regulates $\mathrm{COX}-2$ transcription factor by directly targeting and changing $\mathrm{COX}-2$ protein expression, which in turn affects the proliferation of synovial cells. Taken together, these results suggest that miR-758-3p, in joint fluid, may be used as a diagnostic biomarker for patients with KOA.

\section{Acknowledgements}

The authors of the present study would like to thank Dr Bo Zhang from Heze Municipal Hospital (Heze, China) for providing suggestions and instructions.

\section{Funding}

No funding was received.

\section{Availability of data and materials}

The datasets used and/or analyzed during the current study are available from the corresponding author on reasonable request.

\section{Authors' contributions}

ZL and XH designed the present study. ZL, JS and TL performed the experiments. ZL, JS and XH analyzed the data. ZL and $\mathrm{XH}$ interpreted the results and drafted the initial manuscript. ZL, JS and XH confirm the authenticity of all the raw data. All authors have read and approved the final manuscript.

\section{Ethics approval and consent to participate}

The present study was approved by the Ethics Committee of Heze Municipal Hospital (approval no. 20161226-85; Heze, China) and written informed consent was provided by all patients or their families prior to the study start.

\section{Patient consent for publication}

Not applicable.

\section{Competing interests}

The authors declare that they have no competing interests.

\section{References}

1. Szychlinska MA, Trovato FM, Di Rosa M, Malaguarnera L, Puzzo L, Leonardi R, Castrogiovanni P and Musumeci G: Co-expression and co-localization of cartilage glycoproteins CHI3L1 and lubricin in osteoarthritic cartilage: Morphological immunohistochemical and gene expression profiles. Int J Mol Sci 17: 359, 2016.

2. Musumeci G: Effects of exercise on physical limitations and fatigue in rheumatic diseases. World J Orthop 6: 762-769, 2015 .

3. Castrogiovanni P, Di Rosa M, Ravalli S, Castorina A, Guglielmino C, Imbesi R, Vecchio M, Drago F, Szychlinska MA and Musumeci G: Moderate physical activity as a prevention method for knee osteoarthritis and the role of synoviocytes as biological key. Int J Mol Sci 20: 511, 2019.
4. Di Rosa M, Castrogiovanni P and Musumeci G: The synovium theory: Can exercise prevent knee osteoarthritis? The role of 'mechanokines', a possible biological key. J Funct Morphol Kinesiol 4: 11, 2019.

5. Abbasi J: Can exercise prevent knee osteoarthritis? JAMA 318: 2169-2171, 2017.

6. Koh YG, Jo SB, Kwon OR, Suh DS, Lee SW, Park SH and Choi YJ: Mesenchymal stem cell injections improve symptoms of knee osteoarthritis. Arthroscopy 29: 748-755, 2013.

7. Yuan C, Pan Z, Zhao K, Li J, Sheng Z, Yao X, Liu H, Zhang X, Yang Y, Yu D, et al: Classification of four distinct osteoarthritis subtypes with a knee joint tissue transcriptome atlas. Bone Res 8: 38, 2020.

8. Geng R, Xu Y, Hu W and Zhao H: The association between MMP-1 gene rs1799750 polymorphism and knee osteoarthritis risk. Biosci Rep 38: BSR20181257, 2018.

9. Matsuzaki T, Alvarez-Garcia O, Mokuda S, Nagira K, Olmer M, Gamini R, Miyata K, Akasaki Y, Su AI, Asahara H and Lotz MK: FoxO transcription factors modulate autophagy and proteoglycan 4 in cartilage homeostasis and osteoarthritis. Sci Transl Med 10: eaan0746, 2018.

10. Abramson SB: Nitric oxide in inflammation and pain associated with osteoarthritis. Arthritis Res Ther 10 (Suppl 2): S2, 2008.

11. Shi H, Sun X, Kong A, Ma H, Xie Y, Cheng D, Wong CKC, Zhou Y and Gu J: Cadmium induces epithelial-mesenchymal transition and migration of renal cancer cells by increasing PGE2 through a cAMP/PKA-COX2 dependent mechanism. Ecotoxicol Environ Saf 207: 111480, 2021.

12. Riba A, Deres L, Sumegi B, Toth K, Szabados E and Halmosi R: Cardioprotective effect of resveratrol in a postinfarction heart failure model. Oxid Med Cell Longev 2017: 6819281, 2017.

13. Ogata S, Kubota Y, Satoh S, Ito S, Takeuchi H, Ashizuka M and Shirasuna $\mathrm{K}: \mathrm{Ca}^{2+}$ stimulates $\mathrm{COX}-2$ expression through calcium-sensing receptor in fibroblasts. Biochem Biophys Res Commun 351: 808-814, 2006.

14. Salvado MD, Alfranca A, Escolano A, Haeggström JZ and Redondo JM: COX-2 limits prostanoid production in activated HUVECs and is a source of PGH2 for transcellular metabolism to PGE2 by tumor cells. Arterioscler Thromb Vasc Biol 29: 1131-1137, 2009.

15. Cianchi F, Cortesini C, Schiavone N, Perna F, Magnelli L, Fanti E, Bani D, Messerini L, Fabbroni V, Perigli G, et al: The role of cyclooxygenase- 2 in mediating the effects of histamine on cell proliferation and vascular endothelial growth factor production in colorectal cancer. Clin Cancer Res 11: 6807-6815, 2005.

16. Zheng Y, Comaills V, Burr R, Boulay G, Miyamoto DT, Wittner BS, Emmons E, Sil S, Koulopoulos MW, Broderick KT, et al: COX-2 mediates tumor-stromal prolactin signaling to initiate tumorigenesis. Proc Natl Acad Sci USA 116: 5223-5232, 2019.

17. Hashemi Goradel N, Najafi M, Salehi E, Farhood B and Mortezaee K: Cyclooxygenase-2 in cancer: A review. J Cell Physio 234: 5683-5699, 2018.

18. Yu T, Lao X and Zheng H: Influencing COX-2 activity by COX related pathways in inflammation and cancer. Mini Rev Med Chem 16: 1230-1243, 2016.

19. Yang Q, Fu S and Wang J: Hepatitis C virus infection decreases the expression of Toll-like receptors 3 and 7 via upregulation of miR-758. Arch Virol 159: 2997-3003, 2014.

20. Yao Y, Li Q, Gao P, Wang W, Chen L, Zhang J and Xu Y: Glucagon-like peptide-1 contributes to increases ABCA1 expression by downregulating miR-758 to regulate cholesterol homeostasis. Biochem Biophys Res Commun 497: 652-658, 2018.

21. Mandolini C, Santovito D, Marcantonio P, Buttitta F, Bucci M, Ucchino S, Mezzetti A and Cipollone F: Identification of microRNAs 758 and $33 \mathrm{~b}$ as potential modulators of ABCA1 expression in human atherosclerotic plaques. Nutr Metab Cardiovasc Dis 25: 202-209, 2015.

22. Chen J, Xu Z, Yu C, Wu Z, Yin Z, Fang F and Chen B: miR-758-3p regulates papillary thyroid cancer cell proliferation and migration by targeting TAB1. Pharmazie 74: 235-238, 2019.

23. Hummel R, Wang T, Watson DI, Michael MZ, Van der Hoek M, Haier J and Hussey DJ: Chemotherapy-induced modification of microRNA expression in esophageal cancer. Oncol Rep 26: 1011-1017, 2011.

24. Carrión-Barberà I, Salman-Monte TC, Vílchez-Oya F and Monfort J: Neuropsychiatric involvement in systemic lupus erythematosus: A review. Autoimmun Rev 20: 102780, 2021.

25. Livak KJ and Schmittgen TD: Analysis of relative gene expression data using real-time quantitative PCR and the 2(-Delta Delta C(T)) method. Methods 25: 402-408, 2001. 
26. Manferdini C, Paolella F, Gabusi E, Silvestri Y, Gambari L, Cattini L, Filardo G, Fleury-Cappellesso S and Lisignoli G: From osteoarthritic synovium to synovial-derived cells characterization: Synovial macrophages are key effector cells. Arthritis Res Ther 18: 83, 2016.

27. Joseph T, Zalenskaya IA, Sawyer LC, Chandra N and Doncel GF: Seminal plasma induces prostaglandin-endoperoxide synthase (PTGS) 2 expression in immortalized human vaginal cells: Involvement of semen prostaglandin E2 in PTGS2 upregulation. Biol Reprod 88: 13, 2013.

28. Wu Y, Lin Z, Yan Z, Wang Z, Fu X and Yu K: Sinomenine contributes to the inhibition of the inflammatory response and the improvement of osteoarthritis in mouse-cartilage cells by acting on the Nrf2/HO-1 and NF- $\mathrm{KB}$ signaling pathways. Int Immunopharmacol 75: 105715, 2019.

29. Roos EM and Arden NK: Strategies for the prevention of knee osteoarthritis. Nat Rev Rheumatol 12: 92-101, 2016.

30. Price AJ, Alvand A, Troelsen A, Katz JN, Hooper G, Gray A, Carr A and Beard D: Knee replacement. Lancet 392: 1672-1682, 2018.

31. Amin AR, Dave M, Attur M and Abramson SB: COX-2, NO, and cartilage damage and repair. Curr Rheumatol Rep 2: 447-453, 2000.

32. Tsuge K, Inazumi T, Shimamoto A and Sugimoto Y: Molecular mechanisms underlying prostaglandin E2-exacerbated inflammation and immune diseases. Int Immunol 31: 597-606, 2019.

33. Echizen K, Hirose O, Maeda Y and Oshima M: Inflammation in gastric cancer: Interplay of the COX-2/prostaglandin E2 and Toll-like receptor/MyD88 pathways. Cancer Sci 107: 391-397, 2016.

34. Fabian MR, Sonenberg N and Filipowicz W: Regulation of mRNA translation and stability by microRNAs. Annu Rev Biochem 79: 351-379, 2010
35. Song T, Hou X and Lin B: MicroRNA-758 inhibits cervical cancer cell proliferation and metastasis by targeting HMGB3 through the WNT/ $\beta$-catenin signaling pathway. Oncol Lett 18 : 1786-1792, 2019.

36. Ren J, Yang M, Xu F and Chen J: MicroRNA-758 inhibits the malignant phenotype of osteosarcoma cells by directly targeting HMGA1 and deactivating the Wnt/ $\beta$-catenin pathway. Am J Cancer Res 9: 36-52, 2019.

37. Li J and You X: MicroRNA-758 inhibits malignant progression of retinoblastoma by directly targeting PAX6. Oncol Rep 40: 1777-1786, 2018.

38. Zhang Y and Zhao F: MicroRNA-758 inhibits tumorous behavior in tongue squamous cell carcinoma by directly targeting metadherin. Mol Med Rep 19: 1883-1890, 2019.

39. Zhou GH, Lu YY, Xie JL, Gao ZK, Wu XB, Yao WS and Gu WG: Overexpression of miR-758 inhibited proliferation, migration, invasion, and promoted apoptosis of non-small cell lung cancer cells by negatively regulating HMGB. Biosci Rep 39: BSR20180855, 2019.

40. Lin Z, Fu C, Yan Z, Wu Y, Zhan J, Lou Z, Liao X and Pan J: The protective effect of hesperetin in osteoarthritis: An in vitro and in vivo study. Food Funct 11: 2654-2666, 2020.

This work is licensed under a Creative Commons Attribution-NonCommercial-NoDerivatives 4.0 International (CC BY-NC-ND 4.0) License. 